\title{
Measurement of Moisture Spatial Profiles in Transformer Pressboard
}

\author{
A. V. Manishev, Y. Du, B. C. Lesieutre, and M. Zahn \\ Massachusetts Institute of Technology \\ Department of Electrical Engineering and Computer Science \\ Laboratory for Electromagnetic and Electronic Systems \\ Cambridge, MA 02139, U.S.A.
}

\begin{abstract}
Moisture concentration was measured in a $1.5 \mathrm{~mm}$ thick transformer pressboard as a function of time and position during moisture diffusion process. A flexible interdigital three-wavelength sensor was used to provide the necessary information regarding pressboard dielectric properties at different depths from the sensorpressboard interface. Results were obtained by processing the signals from the three-wavelength sensor, air humidity sensor, and thermocouple installed in the experimental chamber. Air-pressboard equilibrium curves were used to provide boundary conditions and the relative moisture level magnitude. The proposed technology has significant potential for measurement of diffusion processes in various dielectrics.
\end{abstract}

\section{Introduction}

The interdigital frequency-wavenumber dielectrometry methodology is based on excitation of several sets of spatially periodic interdigitated electrodes with a sinusoidal voltage over a wide frequency range [1]. By varying the spatial periodicity of interdigital electrodes, it is possible to vary the depth of penetration of the generated fringing electric fields into the dielectric medium.

An important application of this technology that is currently under investigation is the ability to study moisture dynamic processes in power transformers in order to prevent failures due to free water formation, excessive losses, and dielectric breakdown induced by flow electrification [2]. The preliminary measurements that demonstrate the response of the three-wavelength sensor to the molecular diffusion process were presented in [3].

The goal of this study is to continue the development of interdigital dielectrometry sensors and parameter estimation algorithms to measure the spatial profiles of dynamically varying moisture concentration across the thickness of transformer pressboard in a controlled en- vironment. In this paper, the moisture profiles are calculated assuming a nearly linear relationship between the sensor transcapacitance and the average amount of moisture in the adjacent pressboard volume [4].

\section{Three-wavelength Sensor}

The top view of the sensor used in this study is shown in Figure 1. It consists of three sets of topologically identical interdigital electrodes etched on a common flexible substrate. The gray shaded area indicates guard backplanes on the reverse side of the substrate. The highly hydrophobic Teflon ${ }^{\circledR}$ substrate is $254 \mu \mathrm{m}$ thick and has a relative dielectric permittivity $\varepsilon_{r}$ of 2.1 . The transconductance and transcapacitance between electrode combs are measured by driving one of them with a sinusoidal voltage signal.

In our normal practice, the frequency of this signal varies between $0.005 \mathrm{~Hz}$ and $10 \mathrm{kHz}$. Such a frequency sweep takes up to two hours. In order to monitor the diffusion process with sufficient resolution in time, only selected frequency data points were measured and recorded in this experiment. Attention in the following sections is concentrated on the $1.0 \mathrm{~Hz}$ frequency measurements, Measurements at other frequencies produce similar results in terms of moisture distribution and provide additional confidence in the proposed approach.

\section{Measurement Setup}

A controlled-environment experimental chamber was built to simulate the temperature, moisture, and materials of a power transformer environment [3]. A number of diffusion studies have been performed in this chamber to date. The reported experiment did not involve transformer oil, instead, the ambient air inside the chamber was humidified to a pre-selected value by circulating the moist air through a system of interconnected ducts.

Figure 2 shows the critical part of the experimental setup. A piece of $1.5 \mathrm{~mm}$ thick oil-free transformer 


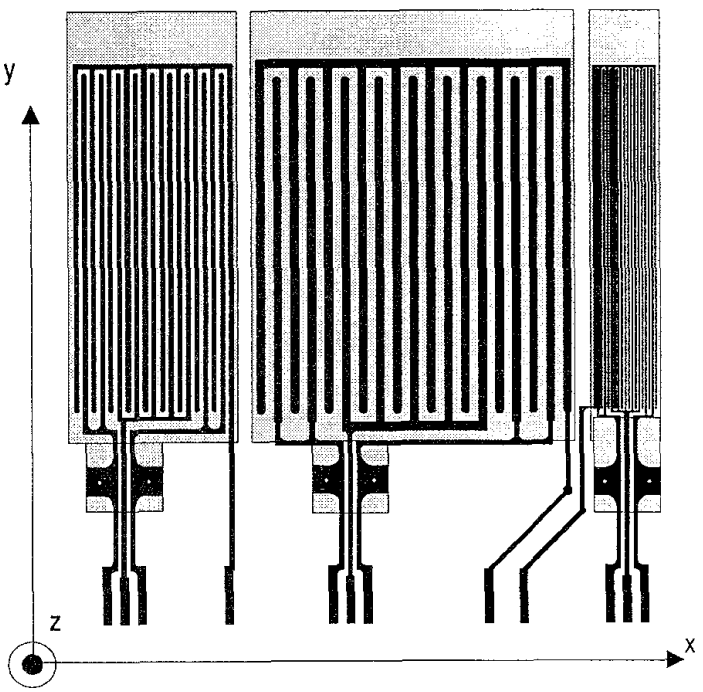

Figure 1: The three-wavelength interdigital sensor with spatial periodicity of $2.5 \mathrm{~mm}, 5.0 \mathrm{~mm}$, and $1.0 \mathrm{~mm}$.

pressboard was positioned next to the sensor. The edges of the pressboard sample were sealed with silicon glue to prevent moisture diffusion from the sides. A number of holes in the metal compression plate provided a path for the moisture molecules to enter the pressboard from the left side (according to the diagram in Figure 2).

Even though the change of the pressboard properties through its thickness is gradual in the discussed experiment, the pressboard in the model is represented as a three-layer medium with each layer thickness corresponding to the effective penetration depth of $0.3 \lambda$ for each wavelength $\lambda$ of the sensor. In other words, the number of wavelengths limits the spatial resolution of the sensor, and only three regions can be a distinctively identified with the three wavelength sensor of Figure 1. Later in this paper, the knowledge of the temporal dynamics of the process helps to improve this representation when plotting moisture profiles.

\section{Experimental Results}

Initially, the pressboard was vacuum-dried. The moisture diffusion process started with a step change in the ambient air humidity from $0 \%$ to about $12 \%$ with the temperature being held at $70^{\circ} \mathrm{C}$. The air-pressboard equilibrium relationships [5] were used to provide boundary conditions at the air-pressboard interface and relative moisture level information needed for the pa-

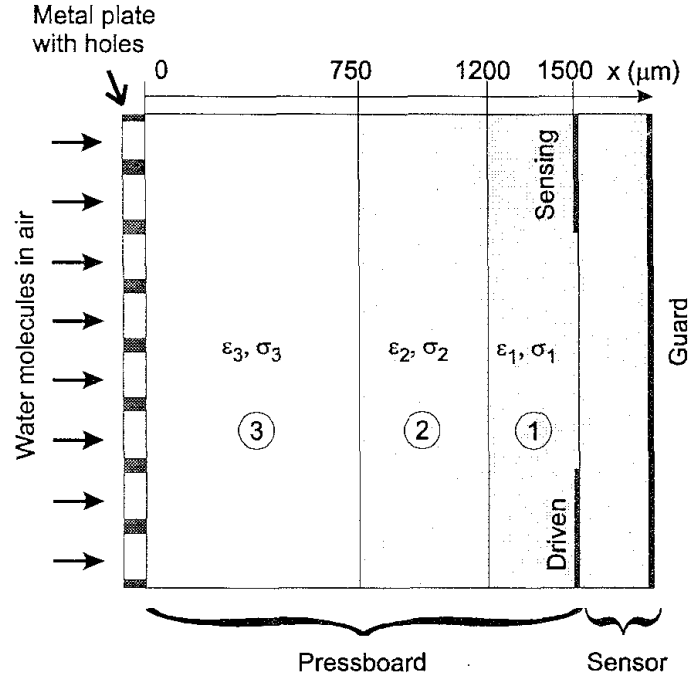

Figure 2: A schematic representation of the experimental arrangement. The pressboard is modeled as a three layer medium with distinct properties for each layer related to the quantity of moisture in each layer.

rameter estimation algorithms. According to these relationships, a $12 \%$ humidity level in air at $70^{\circ} \mathrm{C}$ corresponds to $1.8 \%$ equilibrium moisture concentration in the transformer pressboard. Thus, the left side boundary condition at $x=0$ for this experiment is that the moisture level is equal to $1.8 \%$ for the duration of the entire experiment.

The signals from the sensing electrode were recorded at three frequencies: $0.1 \mathrm{~Hz}, 1 \mathrm{~Hz}$, and $10 \mathrm{~Hz}$. This paper utilizes the signals taken at the frequency of $1 \mathrm{~Hz}$. Figure 3 shows the values of measured transcapacitance for each of the three electrode pairs. Each signal follows approximately the same pattern dictated by the diffusion dynamics. After a small initial delay, the signal grows at a high rate, after which it flattens off, indicating the end of the diffusion process experiment. The initial delay time is the smallest for the largest wavelength because it can "see" deepest into the pressboard.

The relative amplitude of change of transconductance is normally larger than that of the transcapacitance due to the high electric conductivity growth with the increase of the moisture concentration. Nevertheless, as demonstrated below, the transcapacitance provides sufficient sensitivity for the moisture profile measurements. 


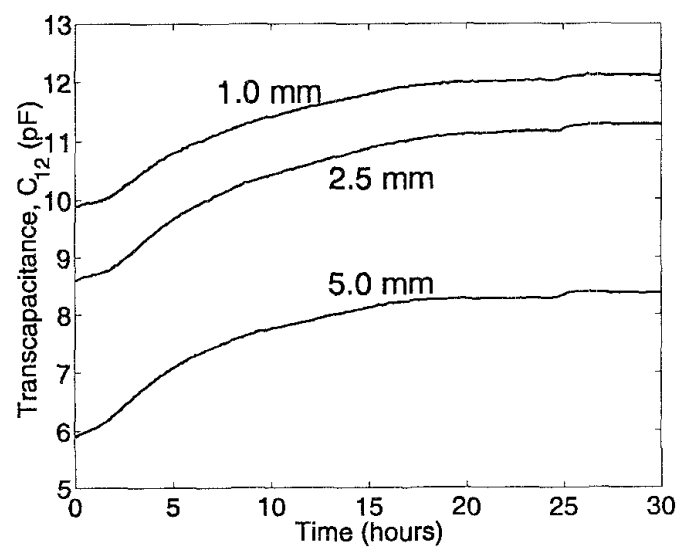

Figure 3: Measured capacitance between each electrode pair throughout the moisture diffusion experiment for each sensor wavelength.

\section{Moisture Profiles}

The transcapacitance signals in Figure 3 were used to generate the curves of moisture concentration in the pressboard layers, assuming a linear relationship between the capacitance values and the moisture levels at each moment of time. That is, the zero level of moisture concentration corresponds to the lowest capacitance value in Figure 3 for each wavelength. The highest capacitance value for each wavelength corresponds to the moisture level of $1.8 \%$. In both cases, at the initial time point and the final time point, the moisture profile is believed to be flat at $0 \%$ and $1.8 \%$, respectively. Consequently, the intermediate values of the moisture concentration would correspond to flat moisture profiles that extend from the left side boundary into the pressboard until the penetration depth interface that is reached. Points to the right of the penetration depth interface would have $0 \%$ moisture until the penetration depth interface reaches $x=1500 \mu \mathrm{m}$.

Figure 4 shows the change in the average values of moisture concentration in the three regions that correspond to the penetration depths of the three wavelengths of the sensor. The average value corresponds to the cumulative amount of moisture in all regions within the reach of a given penetration depth. The fact that the difference of the moisture concentration measured by the $2.5 \mathrm{~mm}$ and the $1.0 \mathrm{~mm}$ wavelengths is very small is related to a relatively small signal delay difference in the detection time analysis. Since the right side blocked boundary condition eliminates the moisture escape, the gradient-driven diffusion process results in a nearly flat moisture concentration profile across the regions 1 and 2 of the pressboard indicated in Figure 2.

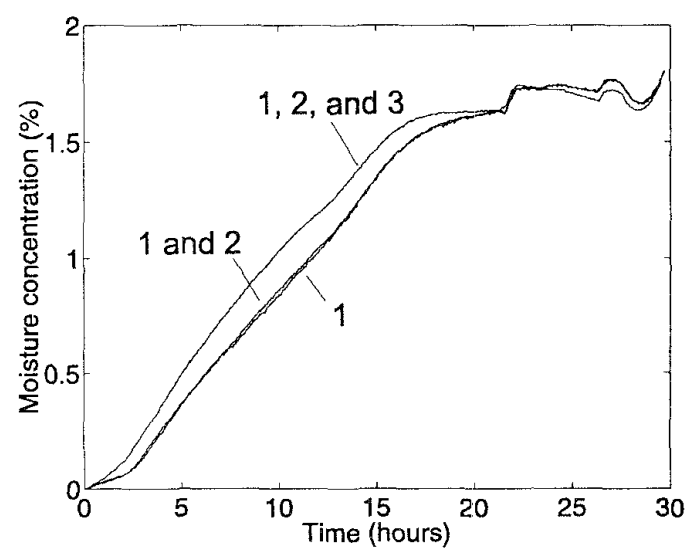

Figure 4: Average moisture level measured by each wavelength: region $1(1 \mathrm{~mm})$, regions 1 and $2(2.5$ $\mathrm{mm})$, and regions 1,2 , and $3(5 \mathrm{~mm})$.

Figure 5 shows calculated moisture profiles from dielectrometry measurements for 14 distinct moments of time. The moisture spatial profiles were calculated from the measurement data using multi-variable parameter estimation algorithms for the three-wavelength sensor combined with the moisture measurement data of the ambient environment together with moisture equilibrium curves.

The process starts with a step change in moisture concentration to $1.8 \%$ at the left boundary at time zero. The first two profiles (at the time moments of two hours and four hours) show a slightly overestimated amount of moisture in the middle region of the pressboard. Future work will include the mass conservation requirements in order to improve the spatial resolution without adding more electrode pairs of different spatial periodicity.

The experiment is terminated after about 30 hours, when the signals essentially stop changing. At this point, the moisture profile is expected to be almost uniform and at the maximum value of $1.8 \%$. The profile at 28 hours has these characteristics.

Now, it is possible to evaluate the moisture diffusion coefficient from the moisture dynamics presented in Figure 5. By simulating the diffusion process using a standard finite difference technique, one can match the measured profiles. The estimated value of the diffusion coefficient is $D=2.3 \times 10^{-11} \mathrm{~m}^{2} / \mathrm{s}$. Figure 6 shows theoretical moisture profiles generated using the estimated value of diffusion coefficient that are in reasonable agreement with the measured profiles in Figure 5. 


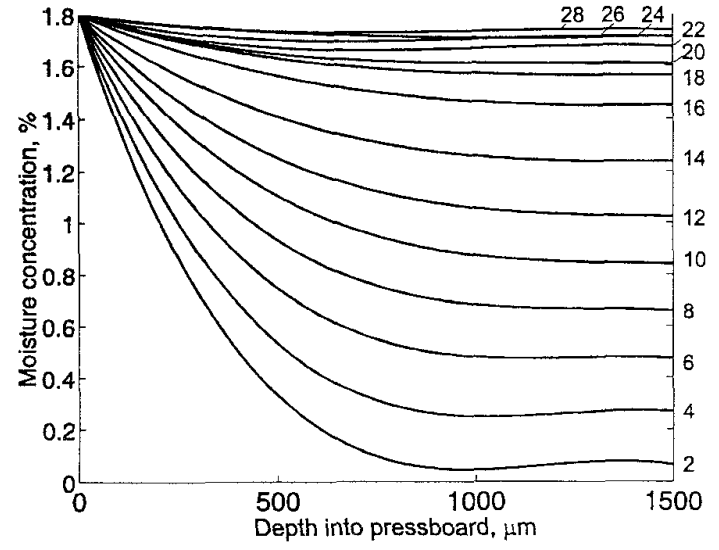

Figure 5: Calculated spatial profiles of moisture concentration across the thickness of pressboard from dielectrometry measurements. Numbers next to the curves correspond to the time in hours at which the profile was measured.

\section{Conclusions}

Real-time on-line measurement of the moisture diffusion process is achieved in this research project. For the first time, the time evolution of the moisture spatial profile in transformer pressboard has been estimated nondestructively. The moisture diffusion profiles have been obtained using the three-wavelength interdigital sensor with a fast algorithm suitable for on-line monitoring with real time display of results. The understanding of moisture-related processes in power transformers can be enhanced with this methodology.

Future experiments will combine a transformer oil convective flow facility and the interdigital sensor setup for studying temperature and moisture transients in a transformer environment. After that, the multi-wavelength sensor could be installed in the low field region of an operating transformer.

\section{Acknowledgments}

The authors would like to acknowledge the support of the Electric Power Research Institute, under grant WO 8619-01, managed by Mr. S. Lindgren, and the National Science Foundation under grant No. ECS-9523128. The donation of Maxwell software by Ansoft Corp. is gratefully appreciated. Financial support through the APPA DEED scholarship to Y. Du and A. V. Mamishev and the Link Foundation Fellowship to A. V. Mamishev is gratefully acknowledged. The authors would like to thank MIT undergraduate student Andrew Taka-

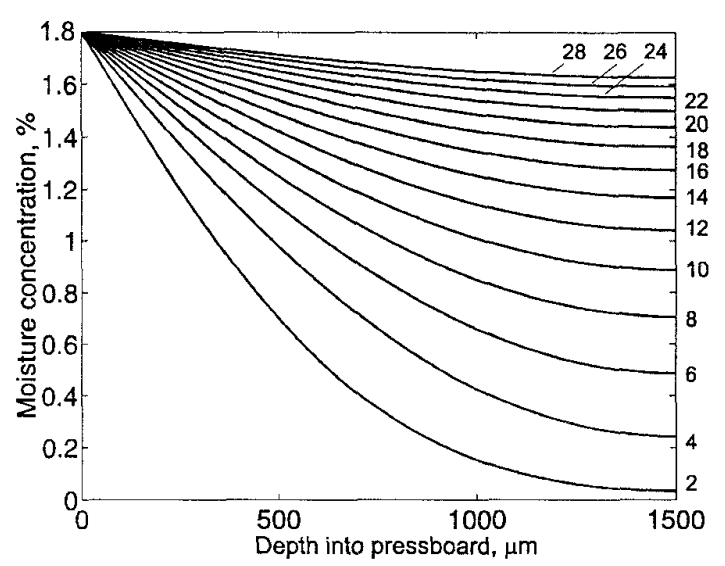

Figure 6: Theoretical spatial profiles of moisture concentration across the thickness of pressboard. Numbers next to the curves correspond to the time in hours after the moisture was stepped on to $1.8 \%$ at $x=0$.

hashi for assistance with data processing done as a part of the MIT Undergraduate Research Opportunities Program and an NSF Research Experiences for Undergraduates grant.

\section{References}

[1] M. C. Zaretsky, L. Mouayad, and J. R. Melcher, "Continuum properties from interdigital electrode dielectrometry," IEEE Transactions on Electrical Insulation, vol. 23, pp. 897-917, Dec. 1988.

[2] Y. K. Sheiretov and M. Zahn, "Dielectrometry measurements of moisture dynamics in oil-impregnated pressboard," IEEE Transactions on Dielectrics and Electrical Insulation, vol. 2, pp. 329-351, June 1995.

[3] Y. Du, B. Lesieutre, and M. Zahn, "Dielectrometry measurements of effects of moisture and anti-static additive on transformer board," in IEEE Conference on Electrical Insulation and Dielectric Phenomena, (Minneapolis, MN), pp. 226-229, Oct. 1997.

[4] A. V. Mamishev, Y. Du, B. C. Lesieutre, and M. Zahn, "Measurement of stratified distributions of dielectric properties and dependent physical parameters," in Fall Meeting of Materials Research Society, (Boston, MA), Dec. 1997.

[5] R. Jeffries, "The sorption of water by cellulose and eight other textile polymers," Journal of the Textile Institute Transactions, vol. 51, no. 9, pp. 339-374, 1960. 